\title{
SAMPLING AND ANALYSIS OF BIOLOGICAL AEROSOLS*
}

\author{
Harriet A. Burge† and William R. Solomon \\ Department of Internal Medicine, University of Michigan, Ann Arbor, MI 48109, U.S.A.
}

(First received $22 \mathrm{July}$ 1985, in final form 28 October 1985 and received for publication $30 \mathrm{July}$ 1986)

\begin{abstract}
The extreme particle size range and enormous heterogeneity of airborne biological particles make sampling a significant challenge. Three major sampler types available include gravity devices, impactors and suction samplers. Gravity methods, while most commonly used, are neither qualitatively or quantitatively accurate and of very limited use. Impaction samplers (rotating, centrifugal) accelerate air by rotating the collecting surface or with a lan. Particles are collected from measured volumes of air but these devices preferentially sample particles larger than $10 \mu \mathrm{m}$. Suction samplers, which efficiently collect particles of a wide size range from measured volumes of air, include slit samplers, cascade impactors, filtration devices and liquid impingers. Suction samplers can retrieve viable particles by direct impaction on culture media, or by subsequent culture of impinger fluid or filter eluates. Nonviable particles can often be identified by microscopic examination of slides, filters or filtrates of impinger fluids. Immunoassays and biochemical assays can be used with impinger fluid and filter eluates to assess antigen and toxin levels in measured air samples.
\end{abstract}

Key word index: Air samplers, bioaerosols, bacteria, fungi, microbial pollution.

\section{INTRODUCTION}

Biological air pollution was first postulated in 55 B.C. when Lucretius concluded that the 'atoms' he saw dancing in a beam of sunshine were carriers of pestilence (Gregory, 1973). Following this astoundingly perceptive observation, it was not until 1873 that serious indoor biological air sampling was undertaken. At that time Cunningham collected air samples in a jail where cholera was rife, while attempting to detect the cause of the disease. He found many fungus spores and pollen grains but no correlation between particle levels and disease rates (Gregory, 1973). Mr Cunningham's inability to isolate and quantify the cholera organism might have been predicted, given its water-borne transmission and his failure to recognize basic principles of bioaerosol sampling. Presented here is a summary of these principles and a survey of currently useful methods available for the study of airborne biological particulates, especially in the indoor environment.

Biologically derived particles that are known to become airbornc in enclosed spices include viruses, which are considerably less than $1 \mu \mathrm{m}$ in diameter, fungus spores, which may exceed $200 \mu \mathrm{m}$, and a wide variety of diflerently sized particles that fall between these extremes (Fig. 1). In addition, biologically derived volatile substances may accumulate in interiors and reach levels sufficient to calusc distatse. This

- Partially supported by grant No. Al10181, U.S. Public Health Service, NIAID.

t All correspondence should be addressed to: Harriet A Burge, R6621 Kresge 1, Box 0529, University of Michigan, Ann Arbor, MI 48109, U.S.A. extreme particle size range, combined with enormous qualitative heterogeneity, make bioaerosol sampling a significant challenge.

\section{SAMPLERS}

Available bioaerosol samplers fall into three general categories: gravity samplers, inertial impactors and filtration devices (Lodge and Pate, 1971). While electrostatic precipitators, which rely on gradient sampling, are commonly used to remove particles (including those of biological origin) from the air, they are rarely used as samplers for biological agents and will not be considered here.

Gravity sampling (as used by biologists) combines both gravitational fallout and inertial processes. Particles are allowed to fall onto some collecting surface (usually either adhesive-coated glass or culture plates). Because air in nature is never still, gravity may play a relatively small part in these collections. In effect, a 'gravity' slide or plate is a constantly changing inertial collector with a gravity component that varies inversely with wind speed and turbulence. Collection efficiency depends on particle size as well as changing levels of atmospheric motion. It is apparent from Fig. 2 that gravity sampling produces both qualitative and quantitative errors in defining patterns of collected bioaterosols. Cunningham 's studies reflected this major deficit: his sampling method was grossly inadequate to efficiently collect particles in the relatively small size range of Vibrio cholera. In his studies, as with all gravity collections, large and often relatively innocuous spores were overestimated in comparison to the smaller and medically more important organisms. In 


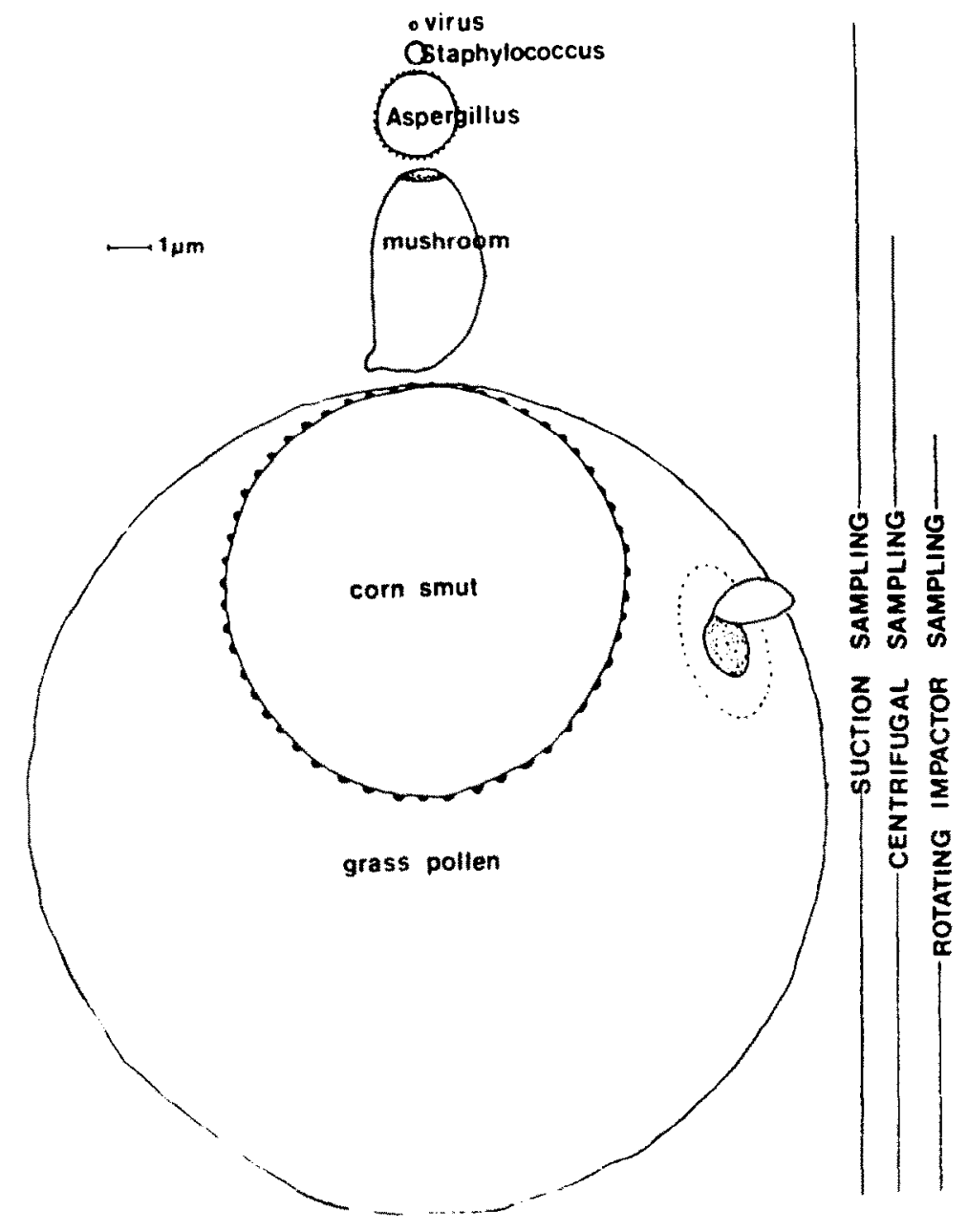

Fig. 1. Diagrammatic representation of the size range of airborne biological particulates and optimum efficiency ranges for three sampling modalities.

addition to these deficits, methods relying on gravity and uncontrolled inertial impaction never provide volumetric data (i.e. data per unit volume of sampled air). In (act, it is only under constant wind conditions that even gross qualitative comparisons among such samples are possible. Unfortunately, this is still the sampling method in most common use today for bioacrosols (Solomon, 1984).

Inertial impactors rely on particle motion for collec. tion, but operate, effectively, under conditions of constant wind speed either by moving collection surfaces through the air at constant speed or by moving air across a stationary collecting surface using a fan or suction source. Included in this category are rotating impactors, liquid impingers and suction impactors.

The collection efficiency of rotating impactors (e.g. the Rotorod sampler, Ted Brown Assoc., Palo Alto, CA) varies directly with particle size, with smaller particles progressively tending to follow the diverted air stream around each collection surface (Edmonds, 1972). Calculated efficiencies for the rotorod sampler using 1.2-mm wide plastic ' $\mathrm{I}$ ' rods (the arrangement most commonly used for studying airborne allergens) as well as two less commonly used configurations are shown in Table 1 . When quite narrow collecting surfaces are used, collection efficiency is satisfactory down to $10 \mu \mathrm{m}$ which includes all pollen types and a wide variety of fungus spores, including many allergen carriers. However, most human pathogens are not efficiently collected by these devices.

Liquid impingers draw air through a liquid, often with the air stream striking a solid surface submerged beneath the surface. Particles are transferred from air to the liquid phase as the bubbles formed are forcefully disrupted (Raynor, 1979). This method is especially useful for the recovery of soluble materials (e.g. mycotoxins, antigens, endotoxins) and for sampling aerosols of bacteria and viruses that require gentle handling.

Suction impactors useful for collecting indoor bioaerosols include slit samplers (e.g. the Burkard spore trap, Burkard Manufacturing Co., Rickmansworth, England), and cascade impactors (e.g. the Andersen Sampler, Andersen Samplers, 4215-C Wendell, Atlanta, GA 30336). Slit samplers draw measured 


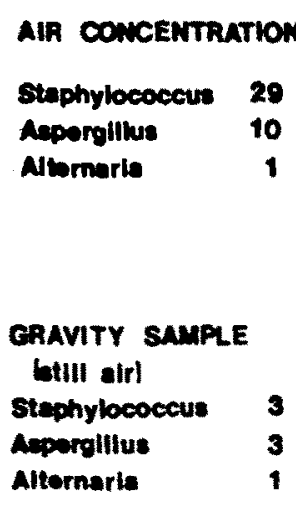

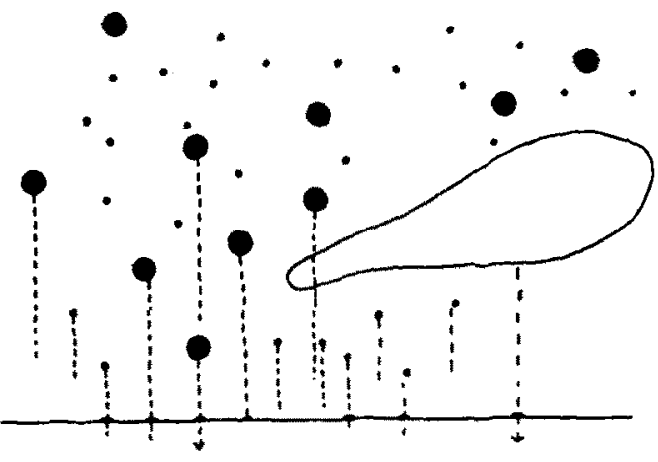

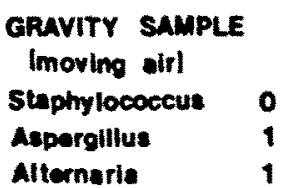

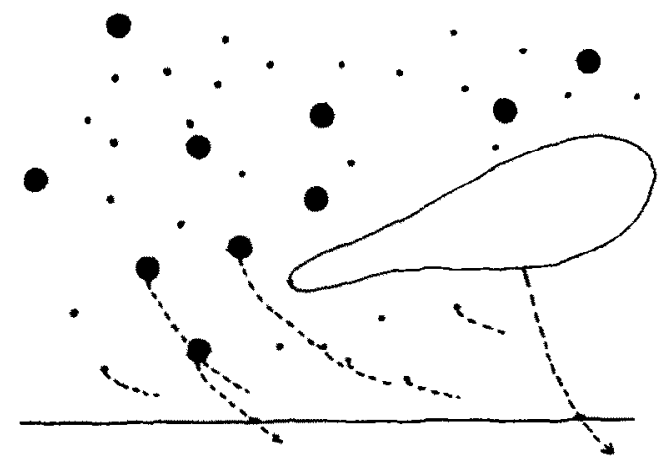

Fig. 2. Demonstration of the inadequacy of gravity bioaerosol sampling in still and moving air.

Table 1. Efficiency $(\%)$ of rotorod sampler with three sampler surface widths (calculated from formulas-see Edmonds, 1972)

\begin{tabular}{lccc}
\hline \multicolumn{4}{c}{ Width of collecting surface $(\mathrm{mm})$} \\
\hline diameter of (spherical) particle & 1.2 & 1 & 0.5 \\
$5 \mu \mathrm{m}$ & $5 \%$ & $5 \%$ & $15 \%$ \\
$10 \mu \mathrm{m}$ & $25 \%$ & $30 \%$ & $80 \%$ \\
$15 \mu \mathrm{m}$ & $70 \%$ & $80 \%$ & $100 \%$ \\
$20 \mu \mathrm{m}$ & $100 \%$ & $100 \%$ & $100 \%$ \\
\hline
\end{tabular}

volumes of air through a narrow orifice (serving to accelerate the air stream), placed close to an adhesivecoated surface or culture plate. The Burkard trap collects particles on a moving drum so that time discriminated samples are possible, enabling continuous (24-h or 7-day) studies of the efrects of various activities on indoor particle levels. Cascade impactors draw air through a relatively broad entrance orifice, then accelerate it stepwise by passage through sieve plates with perforations of smaller and smaller caliber. Impaction is achieved on surfaces placed beneath each sieve platc. Larger particles impact while the air stream is moving relatively slowly, with increasing air speed resulting in the trapping of smaller and smaller particles. As a result, these samplers produce particle size discrimination and have provided evidence that while fungus spores tend to travel as single, 'naked' units, bacteria, which are very small individually, are often dispersed on much larger 'rafts' (droplets or skin scales, for example) (Solomon et al., 1978; Gregory, 1973). All suction devices require that the entrance orifice be directed into the air stream (i.e. be "wind oriented') and that the speeds of air entering the collector and of the wind should be equal. Practically, these conditions are rarely achieved or maintained. Wind alignment is essential, and failure to at least approximately orient the orifice 'into the wind' will result in large losses, especially of larger particles.

While inertial suction samplers are quite efficient for a large range of particle types (provided final air speed is high enough and collection surfaces are sufficiently close to the orifice), some losses do occur. Less than $100 \%$ recovery may result from wall deposition or pass-through, which vary with particle size. Larger particles tend to be trapped on walls; smaller ones are more likely to escape impaction entirely. On the other hand, filtration sampling, which operates on principles of inertial impaction and diffusion as well as sieving. can be $100 \%$ efficient at removing particulates from the air stream, and can be simply achieved with a filter cassette with an appropriate pore-size filter connected to a vacuum line. Since filtration is essentially suction sampling, the cautions regarding orientation of the orifice and concern for wind speed apply. However, once the air with its entrained particles has entered the 
sampler, particle recovery is probably complete to particle diameters well below rated filter pore size (Solomon et al., 1983; Habenicht et al., 1984). Samples collected on filters can be examined microscopically, cultured directly on the filter, or the filters can be washed and eluates examined microscopically, cultured, or analyzed by a wide variety of biochemical and immunological techniques.

\section{SAMPLE ANALYSIS}

There are at least five major methods of sample analysis: culture, direct microscopy, bioassay, biochemical assay and immunological assay. In cultural assays, the collected organisms produce growth that can be identified macroscopically, microscopically or biochemically. These approaches can be used with culture plate collectors (e.g. the Andersen), for processing eluates from filters, and for culturing 'source' samples such as portions of house dust or swabs from contaminated interior surfaces. Cultural assays are especially appropriate for infectious agents such as Legionella, Staphylococcus and invasive fungal pathogens such as Aspergillus fumigatus, where only viable organisms are of interest. Unfortunately, culture plate assays always underestimate actual levels of micro-organisms for several reasons: (1) Some organisms have very specialized growth requirements. Even when a range of culture media are used for each sample to maximize recoveries, some important organisms will not be recorded. Legionella is an example of an important pathogen that cannot be routinely recovered in culture from air. (2) Some micro-organisms produce growth inhibitors that prevent growth of other organisms. (3) Overcrowding may drastically reduce (quantitative) recoveries due to both soluble inhibition factors and 'contact' suppression by adjacent growth points (Blomquist et al., 1984a, b). (4) With filter sampling, dessication and death of fragile micro-organisms during extended collection periods become a significant problem. (5) All organisms have limited viability periods when airborne, and these vary among taxa. At any one time, the fraction of each microbial type that is viable, in a mixed aerosol, may be high or low.

Where viability is not essential in causing disease (e.g. in hypersensitivity conditions), the fact that some organisms cannot be grown becomes an important source of error in estimating exposure conditions. In fact, the impact of each of the variables listed above must be separately quantitated for each organism of interest in planning aerobiological studies. When the organisms to be expected are unknown, our inability to properly assess these sources of error must be recognized as a major limitation in interpreting whatever data result (Burge et al, 1977).

Visual identification of aerosol units is useful when morphologically distinctive particles or, rarely, 'total' fungus spores are of interest. Samples are examined by direct microscopy, identified where possible, and counted. Specialized skills are required to use this method for more than a very limited variety of airborne particle types (Burge et al., 1986). Specific staining (for example using fluorescent antibodies or compounds that adsorb physically to particles) may be useful occasionally where the focus is a single and specific organism for which reagents are available. Possible uses of this approach include monitoring biocontainment facilities, where individual types of bacteria are used for genetic recombination studies.

Methods based on the bioassay of samples involve a living substrate as a prevalence indicator. The Limulus amoebocyte assay, in which this unicellular organism is lysed by bacterial endotoxin in dose dependent fashion, exemplifies this approach. The bioassay used most often by allergists is the direct skin testing of human subjects. In practice, suitably processed filter eluates, impinger fluids, or most frequently, defined extracts of dust, pollens or spores are introduced into the outermost skin layers of people sensitive to specific antigens. Human skin will react to microgram quantities of relevant antigen in this test (Habenicht et al., 1984), providing a useful assay tool.

Biochemical assays are useful for airborne substances such as mycotoxins having well-defined molecular structures and predictable behavior in molecular sieve columns and paper chromatographic systems (Rodericks et al., 1977).

Among the newest analytic methods for bioaerosol sampling are immunological assays such as the enzyme-linked immunosorbent assay (ELISA) and the radio-allergosorbent test (RAST). In these procedures, a known antigen is adsorbed to a solid phase (usually a polystyrene microtiter plate for the ELISA, and paper discs or cellulose beads for the RAST). Human serum with antigen-specific antibodies is added, followed by tagged antibody to human antibody, made in another species. Especially useful in this step are mouse monoclonal anti-human antibodies labelled with an enzyme (e.g. alkaline phosphatase) or a radioactive tag $\left(\mathrm{I}^{125}\right)$. Addition of substrate to the enzyme preparation produces a colored product in amounts proportional to the binding of human serum antibody to the solid phase antigen (Sepulveda et al., 1979). When radioisotopes are used in related systems, bound radioactivity rises as the serum antibody bound increases. If soluble antigen (either known, homologous or an unknown sample) is added to the human scrum before the assay is run, homologous antigen, if present, will bind to serum antibodies and reduce either final color production or the amount of radioactivity bound. Using this approach, the percent inhibition (of a control assay) will be proportional to the amount of homologous antigen present in the unknown sample (Gleich et al.. 1974). In principle, specific amorphous antigens from free air or sites near suspected sources can be quantified using appropriate materials. In studying building related epidemics, for example, a panel of solid phase antigens associated with hypersensitivity pneumonitis 
or humidifier fever can be used. In homes, one can study concentrations in house dust of cockroach antigen (Sanders et al., 1985), as well as animal-derived sensitizers, and a variety of fungus-related antigens. While established only within the last several years, this assay method may prove to be the most valuable in our arsenal to study both indoor and outdoor air for a wide variety of biological contaminants.

\section{SAMPLING DESIGN}

The choice of a sampler (or samplers) to use in conjunction with specific systems of analysis depends on which bioaerosols deserve primary interest. In surveys directed to specific organisms, a single preferred sampler and an operating mode that will maximize recoveries of that organism or group can often be identified. For bacteria, a liquid impinger or cascade impactor containing growth medium generally is most appropriate. For Allernaria (a readily recognizable larger fungus spore), a slit sampler and direct (visual) identification tends to be the method of choice. However, the usual case in bioaerosol research finds the investigator unsure of which organism(s) and substance(s) may be expected and unable to predict the relative importance of any type. Since there is no single sampler/analysis method that will cover all bases, one is forced to apply several less than perfect approaches. The combined use of slit sampler (visual identification), culture plate cascade samplers in arrays using each of at least three culture media, and either a filter sampler or liquid impinger (facilitated by bioassay, biochemical or immunological analysis) covers a broad range of airborne particle types.

The statistical design of bioaerosol studies remains difficult due to the enormous complexity of most natural aerosols, and large population variances resulting from interacting environmental and sampling factors. Source strength alone is a complex variable inevitably producing non-linear relationships and non-normal population distributions. Prevalence data for air spora are almost never normal and can only rarely be transformed into a semblance of normality (Solomon et al., 1980). Nonparametric statistical methods should therefore be used whenever possible. Fortunately, bioaerosol effects, at least with respect to fungus phytopathogens and other infectious agents, often are of the 'all or nothing' variety so that, with relatively few samples one can determine whether or not serious contamination exists. In general, if more than 10 samples per indoor or outdoor group are necessary to achieve statistical significance, the prevalence differences between these sites will probably be small and not biologically important. This, in fact, often makes it possible to obtain enough samples in one working day to determine whether or not a biopollution problem exists.

\section{CONCLUSION}

Bioaerosol pollution affects a wide variety of people in many ways but has been little studied in indoor situations. Hypersensitivity conditions caused by bioaerosols, as well as airborne epidemics of infectious disease, and exposure to potentially dangerous aerosolized biological products are all factors that make research in indoor biological air pollution important. Energy conservation effects have created indoor environments that not only electively retain released bioaerosols, but may support their production as well. Renewed attention to biological air pollution will require application of efficient, portable samplers that produce readily and multiply analyzable samples. Using samplers of defined capability as well as uniform analytic methods, nationwide background standards need to be established for at least those airborne biological contaminants having recognized adverse health effects. In addition, risk assessment must be made for a broad range of contaminants and upper limit standards progressively established.

\section{REFERENCES}

Blomquist G., Strom G. and Stromquist L. (1984a) Sampling of high concentrations of airborne fungi. Scand. J. Work Envir. Hith 10, 109-113.

Blomquist G., Palmgren U. and Strom G. (1984b) Improved techniques for sampling airborne fungal particles in highly contaminated environments. Scand. J. Work Envir. Hith 10, 253-258.

Burge H. P., Boise J. R., Rutherford J. A, and Solomon W. R. (1977) Comparative recoveries of airborne fungus spores by viable and nonviable modes of volumetric collection. Mycopathologia 61, 27-33.

Burge H., Chapman J. and Jelks M. (1987) Quality Control of Multisource Aeroallergen Data. Grana (in press).

Edmonds R. L. (1972) Collection efficiency of rotorod samplers for sampling fungus spores in the atmosphere. Plamt Dis. Rep. 56, 704-708.

Gleich G. J., Larson J. B., Jones R. T. and Baer H. (1974) Measurement of the potency of allergy extracts by their inhibitory capacities in the radioallergosorbent test. $J$. Alleray Clin. Immunol. 53, 158 169.

Gregory P. H. (1973) The Microbiology of the Aimosphere, pp. 126-143. John Wiley, New York.

Habenicht $H$., Burge $H$. Muilenberg $M$. and Solomon $W$. (1984) Allergen carriage by atmospheric aerosol-1I. Ragweed pollen determinants in submicronic atmospheric fractions. J. Allergy Clin. Immunol. 74, 64-67.

Lodge J. P. and Patc J. B. (1971) Analysis of air for pollutants. In Treatise on Analytical Chemistry (Edited by Koltoll I. M., Elving P. J. and Stross F. H.) Pt. III, Vol. 2., pp. 199-252. John Wiley, New York.

Raynor G. S. (1979) Sampling techniques in Aerobiology. In Aerobiology, The Ecological Systems Approach, pp. 151-172. Dowden, Hutchinson \& Ross, Stroudsburg. PA.

Rodericks I. V., Hesseltine C. W. and Mehiman M. A. (1977) Mycotoxins in Human and Animal Health. Pathotox Publ, Park Forest South, III.

Sanders G., Burge H., Muilen berg M. and Solomon W. (1985) Detection of cock roach antigen in commercial house dust by ELISA inhibition. J. Allergy Clin. Immunol. 75, 166.

Sepulveda R., Longbottom J. and Pepys J. (1979) Enzyme. linked immunosorbent assay (ELISA) for $\mathrm{IgG}$ and $\mathrm{lgE}$ 
antibodies to protein and polysaccharide antigens of Aspergillus fumigatus. Clin. Allergy 9, 359-371.

Solomon W. R. (1984) Sampling airborne allergens. Ann. Allergy 52, 140-147.

Solomon W. R., Burge H. A. and Boise J. R. (1978) Airborne Aspergillus fumigatus outside and within a large clinical center. J. Allergy Clin. Immunol. 62, 56-60.

Solomon W. R., Burge H. A, Boise J. R. and Becker M. (1980) Comparative particle recoveries by the retracting rotorod, rotoslide and Burkard spore trap sampling in compact array. Int. J. Biomet. 24, 107-116.

Solomon W. R., Burge H. A. and Muilenberg M. L. (1983) Allergen carriage by atmospheric aerosol-I. Ragweed polien determinants in smaller micronic fractions. $J$. Allergy Clin. Immunol. 72, 443-447.

Wray B. B. and O'Stcen K. (1975) Mycotoxin-producing fungi from house associated with kukemia. Arch. Envir. Hlth 30, 571-573. 